\title{
LOS XENARTHRAS FÓSILES DE LA LOCALIDAD DE BUENOS AIRES DE PALMARES (BLANCANO TARDÍO - IRVINGTONIANO TEMPRANO), PROVINCIA DE ALAJUELA, COSTA RICA
}

\author{
César Laurito Mora ${ }^{1,2 *}$, Ana Lucía Valerio Zamora ${ }^{3}$, Eduardo Antonio Pérez Gamboa ${ }^{4}$ \\ ${ }^{1}$ INA, Instituto Nacional de Aprendizaje - Núcleo de Turismo \\ ${ }^{2}$ Investigador Asociado-Departamento de Historia Natural, Museo Nacional de Costa Rica \\ Apdo. Postal 203-2200, Coronado; San José - Costa Rica \\ ${ }^{3}$ Departamento de Historia Natural, Museo Nacional de Costa Rica \\ Apartado Postal 749-1000, San José - Costa Rica \\ ${ }^{4}$ Dirección de Geología y Minas, Ministerio del Ambiente y Energía \\ Apartado Postal 62-1009, San José-Costa Rica \\ *Autor para contacto: cesarlaurito@ice.co.cr
}

(Recibido 03/10/06; aceptado 03/11/06)

\begin{abstract}
Two new glyptodonts are described for Costa Rica. The species Glyptotherium cf. arizonae and the dwarf glyptodont Pachyarmatherium leiseyi. This last species which was firstly recorded for the Upper Pliocene of Florida; its finding represents the southernmost record for Central America of these species. Key words: Cingulata, Glyptodontinae, Glyptatelinae, Upper Pliocene, Lower Pleistocene, Biotic Interchange, Costa Rica.

RESUMEN: Dos nuevas especies de gliptodontes se describen para Costa Rica, la especie Glyptotherium cf. arizonae y la forma enana Pachyarmatherium leiseyi, esta última inicialmente descrita para el Plioceno Superior de Florida. Su hallazgo representa el primer y más austral registro fósil de esta especie para América Central. Palabras clave: Cingulata, Glyptodontinae, Glyptatelinae, Plioceno Superior, Pleistoceno Inferior, Intercambio Biótico, Costa Rica.
\end{abstract}




\section{INTRODUCCIÓN}

Los xenarthras son un grupo de mamíferos originados en América del Sur durante el Paleógeno que alcanzó América del Norte al menos en dos oportunidades, la primera vez vía las Antillas hace 9 Ma y la segunda, vía el Istmo de Panamá hace unos 3,5 Ma. Su característica anatómica más relevante es la carencia de esmalte en los dientes o la ausencia de los mismos como en los osos hormigueros.

En la actualidad este grupo está representado por los perezosos, armadillos y osos hormigueros, todos de mediano y pequeño tamaño, a pesar de que muchas formas neógenas y cuaternarias fueron verdaderos gigantes.

En Costa Rica los xenarthras fósiles son poco conocidos y el registro de sus hallazgos se ha limitado a la aparición de unos cuantos osteodermos y restos óseos atribuibles a formas propias del Rancholabreano. Recientemente, Valerio et al. (2005) han descrito la especie Glyptotherium aff. texanum Osborn, 1903, para el Pleistoceno de la provincia de Alajuela. Sin embargo, durante el año 2005, luego de realizar varias prospecciones en el cantón de Palmares se recuperó una muestra de restos óseos diversos en los que se encontraron asociados dos especies de gliptodontes y un gomfotérido que se encuentra en proceso de restauración.

El objetivo del presente trabajo es dar a conocer los nuevos hallazgos paleovertebradológicos y a la luz de estos, interpretar la paleoecología y edad del yacimiento.

El hallazgo se realizó en el distrito de Buenos Aires del cantón de Palmares, provincia de Alajuela, en las coordenadas $10^{\circ} 04^{\prime} 44^{\prime \prime} \mathrm{N} /$ $84^{\circ} 25^{\prime} 58^{\prime}$ 'W; en las cercanías de la quebrada Fierro.

\section{PALEONTOLOGÍA}

A continuación se describen los aspectos sistemáticos y paleoecológicos del hallazgo (Fig. 1).

\section{Sistemática:}

Se sigue la sistemática propuesta por McKenna \& Bell (1997).

Superorden Xenarthra Cope, 1889

Orden Cingulata Illiger 1811

Superfamilia Glyptodontoidea Gray, 1869

Familia Glyptodontidae Gray, 1869

Subfamilia Glyptatelinae Castellanos, 1932

Género Pachyarmatherium Downing \& White, 1995

\section{Pachyarmatherium leiseyi \\ Downing \& White, 1995}

\section{Sinonimia:}

- Pachyarmatherium leiseyi nov. sp. en Downing \& White, 1995, págs. 378-388, figuras 1-4.

- Pachyarmatherium leiseyi en Hulbert, 2001, págs. 178-179, figura 10.4D-F.

\section{Material:}

Un osteodermo aislado y fragmentado, depositado en la colección de fósiles del Museo Nacional de Costa Rica bajo el código CFM1734.

\section{Medidas:}

Altura $10.00 \mathrm{~mm}$ y diámetro aproximado $11.70 \mathrm{~mm}$

\section{Descripción:}

Osteodermo muy grueso, pequeño y roto de contorno presumiblemente hexagonal. Su superficie externa es tersa, presenta un surco radial que se proyecta a partir del vértice que conforma la unión de dos surcos de disposición anular. Los surcos anulares, por lo que se puede observar, configuran una figura central compuesta por un 


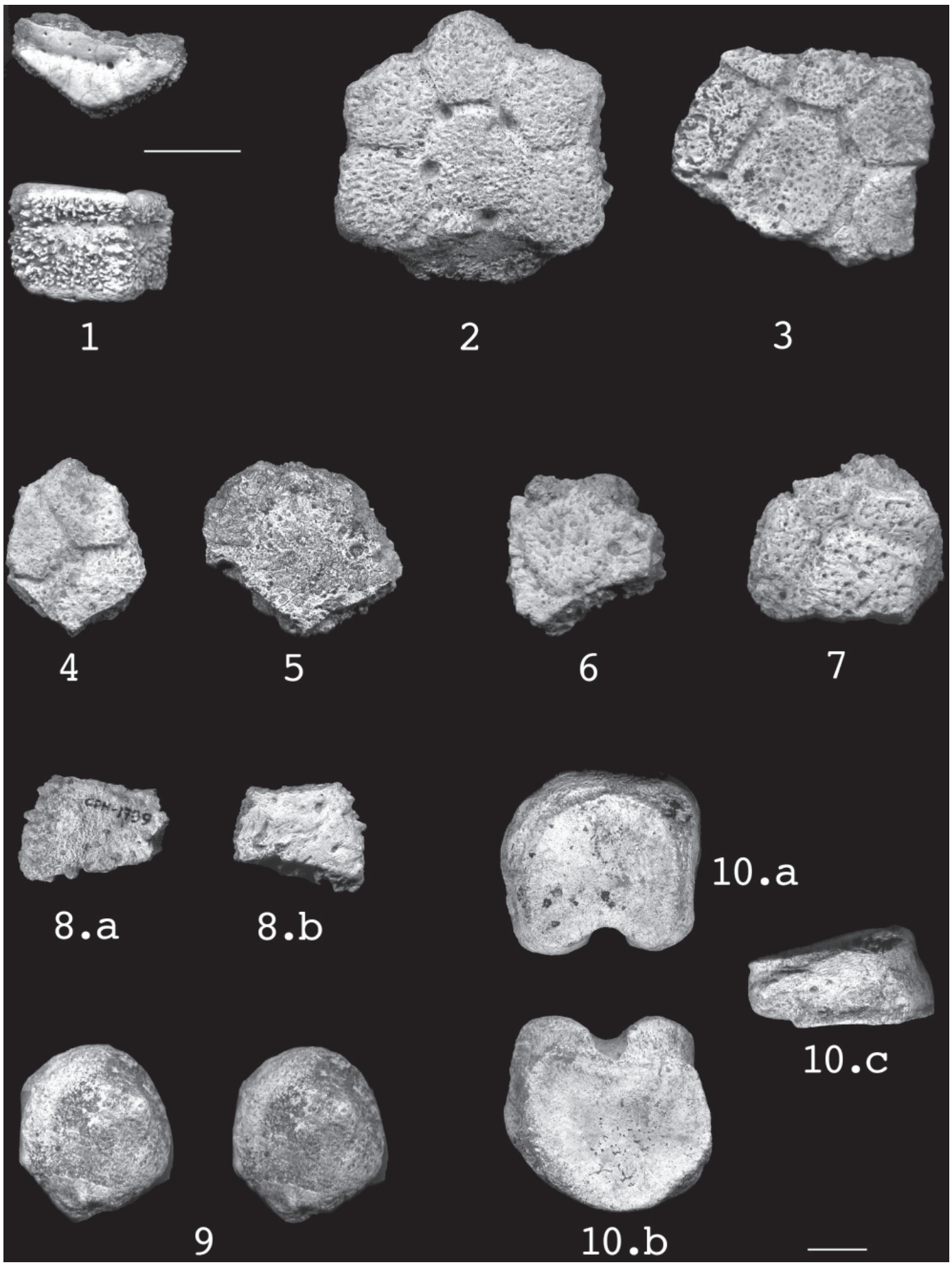

Fig. 1: Pachyarmatherium leiseyi, 2-7 osteodermos de Glyptotherium cf. arizonae; 8. osteodermo marginal; 9, par estereográfico de osteodermo del tubo caudal y 10, falange del tercer dígito del pie izquierdo. escala visual equivalente a $10 \mathrm{~mm}$. 
hexágono excéntrico abierto con forámenes pilosos alineados a lo largo de los surcos; el foramen que se ubica en el vértice de la unión de dos surcos es mucho más grande y conspicuo que los restantes forámenes.

Las caras laterales de la placa son elevadas y sus superficies están constituidas por dos niveles altamente rugosos diferenciados entre si por un nivel intermedio relativamente liso de ancho variable. El nivel rugoso superior alcanza 1,5 mm de espesor y limita con la superficie externa del osteodermo.

\section{Discusión:}

Webb et al. (1989) fueron los primeros en notar la presencia de un pequeño y nuevo glyptodonte en el Pleistoceno temprano de Florida que luego fue formalmente descrito por Downing \& White, 1995 como Pachyarmatherium leiseyi.

Pachyarmatherium leiseyi Downing \& White, 1995 es un gliptodonte de pequeño tamaño, caracterizado por poseer pequeños pero muy gruesos osteodermos pentagonales o hexagonales, con figuras centrales excéntricas, con sistema de surcos periféricos anulares formando hexágonos, en algunos casos abiertos e incompletos.

P. leiseyi Downing \& White, 1995 poseía un caparazón tipo gliptodonte, pero se diferencia de estos por poseer un cinturón móvil en la parte media, condición que recuerda a los dasypódidos, lo que indujo a Downing \& White, 1995 a incluirlo dentro de este grupo. Sin embargo, como señalan Vizcaíno et al., 2003, este es un carácter primitivo de los gliptodontes que está presente en los Propalaeohoplophorinae del Mioceno Temprano de la Patagonia (Scout, W.B. 19031904 en Vizcaíno et al., 2003).

Por otra parte, los osteodermos de Pachyarmatherium son muy similares a los osteodermos de Neoglyptatelus (Carlini et al., 1997); una forma sudamericana ampliamente distribuida desde el sur de Uruguay hasta la localidad de La Venta en Colombia. Por lo cual Vizcaíno et al., 2003, consideran la posibilidad de que Neoglyptatelus sea un sinónimo "junior" de Pachyarmatherium.

El ejemplar costarricense, a pesar de ser muy fragmentario es fácilmente identificable, a causa de su tamaño, grosor y por la disposición de los surcos como en P. leiseyi Downing \& White, 1995.

\section{Distribución paleobiogeográfica y bioestrati- gráfica:}

Pachyarmatherium leiseyi ha sido registrado para el Plioceno Tardío y el Pleistoceno Temprano de Florida (Leisey Shell Pit, Hillsborough County y Haile 16A, Alachua County entre otros) y el Plioceno Tardío de Dorchester County en Carolina del Sur (Downing \& White, 1995 y Hulbert, 2001).

\section{Paleobiología:}

Downing \& White, 1995, sugieren que la dieta de $P$. leiseyi se basaba en la ingestión de termitas y hormigas, lo que deducen de los escasos elementos mandibulares recuperados caracterizados por piezas dentales frágiles y reducidas, y por la posesión de fuertes garras aptas para la excavación de termiteros y hormigueros. Por la similitud con el género "Neoglyptatelus" al cual Kay \& Madden (1997) proponen un hábitat heterogéneo constituido por bosques discontinuos dentro de áreas interfluviales, con pluviosidad anual estimada entre los 1500 y $2000 \mathrm{~mm}$ y temperaturas cálidas, es posible suponer que estas condiciones hallan permitido la dispersión de Pachyarmatherium desde América del Sur hacia Norteamérica.

El hallazgo de esta especie en la localidad de Buenos Aires, permite establecer una edad confiable para los sedimentos lacustres de Palmares, Blancano Tardío o Irvingtoniano Temprano.

Subfamilia Glyptodontinae Gray, 1869

Tribu Glyptotheriini Castellanos, 1953

Género Glyptotherium Osborn, 1903

\section{Glyptotherium cf. arizonae Gidley, 1926}

\section{Sinonimia:}

- Glyptotherium sp. cf. G. arizonae Gidley, 1926 en Gillette \& Ray, 1981- pág. 182, fig.86. 
- Glyptotherium arizonae en Hulbert, 2001, pág. 180, fig. 10.4A.

- Glyptotherium arizonae Gidley, 1926 en Cisneros, 2005-pág. 243, fig. 4A, B, G-J

\section{Material:}

Los especimenes referidos se encuentran depositados en la colección de fósiles del Museo Nacional de Costa Rica, bajo los códigos CFM1733, CFM-1735,1736 (hueso del pes) a CFM1745, CFM-1842, CFM-1844 y CFM 1845.

\section{Descripción:}

Ejemplar CFM-1733, corresponde a un fragmento de osteodermo con superficie externa notablemente rugosa y superficie interna suave con varios forámenes pilosos. Su espesor alcanza los 20,0 mm y una longitud máxima observable de 41,0 mm; a pesar de su estado de preservación, se puede deducir que la figura central tiene un diámetro de $28,0 \mathrm{~mm}$ y la zona marginal es relativamente muy reducida por lo que es posible asociarla a un escudo dérmico interior próximo al borde marginal del caparazón.

Ejemplar CFM-1735, osteodermo fragmentado de tipo roseta de gran tamaño, con superficie externa muy porosa en la que sobresalen conspicuos forámenes pilosos; 17,0 mm de espesor y una figura central poligonal de aproximadamente de $24,0 \mathrm{~mm}$ de diámetro, pétalos marginales preservados parcialmente. De acuerdo a su forma es posible deducir que este osteodermo corresponda con un escudo dérmico interior cercano al borde posterior o anterior del caparazón.

Ejeplar CFM-1737, osteodermo fragmentado del cual solo se preservó la figura central que es circular con diámetro de $17,0 \mathrm{~mm}$ y una pequeña porción de los pétalos marginales, su espesor alcanza los 15,0 mm, su cara interna es liza y cóncava. Como en el caso anterior corresponde a un escudo dérmico interior, cercano al borde posterior o anterior del caparazón.

Ejemplar CFM-1738, fragmento de osteodermo del que solamente se preservó parte de la figura central de contorno hexagonal con 40,0 mm de diámetro aproximado, fuertemente perforada y que correspondía a un escudo dérmico interior de gran diámetro 110 o $120 \mathrm{~mm}$.

Ejemplar CFM-1739, fragmento de placa estrictamente marginal, carente de figura central, superficie externa poco ornamentada, con escasos forámenes pilosos y con un espesor máximo observable $14.80 \mathrm{~mm}$; la superficie del borde marginal es rugosa y pustulada.

Ejemplar CFM-1740, osteodermo interior, roseta fragmentado de gran tamaño, con figura central elíptica y pétalos poligonales separados por amplios surcos radiales y un surco anular, se observan forámenes pilosos de 1,8 mm de diámetro en las convergencias de los surcos radiales con el surco anular.

Ejemplar CFM-1741 fragmento de osteodermo con figura central subcircular de $20,0 \mathrm{~mm}$ de diámetro, rodeada por un amplio surco anular con amplios forámenes pilosos en la unión de los surcos radiales. Este osteodermo se corresponde con un escudo interior.

Ejemplar CFM-1742 osteodermo tipo roseta fragmentado con un espesor máximo observable de $18,0 \mathrm{~mm}$, figura central apenas reconocible. Este osteodermo se corresponde con un escudo interior.

Ejemplar CFM-1744 osteodermo fragmentado de pequeño tamaño con amplia figura central con $20,0 \mathrm{~mm}$ de diámetro sin pétalos marginales y $15,0 \mathrm{~mm}$ de espesor, con borde marginal reducido por lo que se asume corresponde a un escudo dérmico interior próximo al borde marginal del caparazón.

Ejemplar CFM-1842 osteodermo en forma de roseta de gran tamaño casi completo con un diámetro de 52,0 $\mathrm{mm}$ y un espesor de 18,65 $\mathrm{mm}$. El diámetro de la figura central es de $20,0 \mathrm{~mm}$ y el diámetro de los pétalos 11,60 mm, apertura de los forámenes pilosos $3,55 \mathrm{~mm}$.

Ejemplar CFM-1844 osteodermo de anillo caudal de 33,0 mm de diámetro, figura central muy amplia con cono central excéntrico y borde marginal reducido; la cara ventral es cóncava y liza.

Ejemplar CFM-1845 fragmento de osteodermo en forma de roseta, en el que se observa parte de la figura central y fragmentos de tres pétalos.

Ejemplar CFM-1743, corresponde a un fragmento no determinado de hueso plano; los ejemplares CFM-1745, 1746, 2001 y 2018 
corresponden a fragmentos de osteodermos de posición incierta.

Ejemplar CFM-1736, único elemento no constituyente del caparazón encontrado, el mismo corresponde a falange I (proximal) del tercer dígito del pie izquierdo. Su diámetro máximo antero-posterior alcanza los $32,5 \mathrm{~mm}$ y su máximo diámetro transverso en la faceta distal $35 \mathrm{~mm}$.

\section{Discusión:}

Glyptotherium arizonae Gidley, 1926 se caracterizan por poseer placas gruesas, relativamente muy grandes con figura central bastante plana. En comparación con la especie Glyptotherium texanum Osborn, las figuras centrales de $G$. arizonae tienden a ser más grandes que las figuras marginales.

En relación con las placas de G. floridanum, $G$. arizonae se caracteriza por placas muy grandes, incluso algunas duplican el diámetro de la especie de La Florida.

Además, la falange encontrada muestra dimensiones que al ser comparadas con los valores biométricos computados por Gillette \& Ray (1981) para las diferentes especies norteamericanas, caen en el rango de $G$. arizonae.

Por otra parte, es un hecho que las placas de Buenos Aires de Palmares, están asociadas a Pachyarmatherium leiseyi por lo que se descarta su comparación con las formas rancholabreanas mucho más jóvenes.

Teniendo en cuenta las características antes descritas es posible asumir que las placas de Glyptotherium recuperadas son más cercanas morfológicamente a $G$. arizonae que a las restantes especies. Por ello se asume que los restos descritos pertenecen a Glyptotherium sp. cf. G. arizonae Gidley, 1926.

\section{Distribución paleobiogeográfica y bioestratigráfica:}

La especie Glyptotherium arizonae Gidley, 1926 se ha descrito para las localidades del Irvingtoniano de Cochise County en Arizona y Tillman County en Oklahoma, por lo que Gillette \& Ray (1981) le asignan una edad mamífero Irvingtoniano a la especie. Sin embargo, escudos grandes y aislados, atribuidos por Gillette \& Ray (1981) como pertenecientes a Glyptotherium cf. G. arizonae Gidley, 1926 proceden de diversas localidades del Irvingtoniano Temprano de $\mathrm{La}$ Florida como Inglis Fauna 1A; Citrus County y Gilchrist County pero otros proceden de localidades como Charlotte Harbor y Santa Fe River IB, consideradas por Webb, 1974 y MacFadden \& Waldrop, 1980 más antiguas, Blancano Tardío.

De igual forma, Lucas et al. (1993) describen un osteodermo caudal de Glyptotherium procedente de la localidad de Arroyo Tijeras de edad Irvingtoniano Temprano, en Bernalillo County, Nuevo México, que luego Morgan \& Lucas (2003) consideran pertenece ciertamente a G. arizonae Gidley, 1926. Además, estos autores señalan que los fósiles más diagnósticos para la localidad de Sandoval County, también en el Estado de Nuevo México, son cinco osteodermos de $G$. arizonae los que identifican con base en su gran tamaño y espesor y el relativo pequeño tamaño de las figuras centrales que son característicamente planas, la edad atribuida a estos hallazgos por dichos autores es Blancano Tardío.

En América Central G. arizonae Gidley, 1926 ha sido descrita para el Pleistoceno Temprano-Medio de la localidad Río Tomayate en el Salvador por Cisneros (2005).

Teniendo en cuenta lo anterior es posible asumir una edad Blancano Tardío - Irvingtoniano Temprano para los restos de Glyptotherium cf. G. arizonae Gidley, 1926 procedentes de Buenos Aires de Palmares, lo cual es coincidente con la edad de Pachyarmatherium leiseyi Downing \& White, 1995.

\section{Paleobiología:}

Los gliptodontes se originaron en el Neotrópico suramericano (Hoffstetter, 1958) y es muy probable que el género Glyptotherium se diferenció en el norte de América del Sur durante el Plioceno Temprano y alcanzó América Central luego del establecimiento del Istmo de Panamá.

Glyptotherium arizonae Gidley, 1926 fue la especie más grande y voluminosa de entre todos las especies de Glyptotherium descritas hasta ahora, se especula que sus individuos adultos llegaban a pesar más de una tonelada (Kurtén \& Anderson, 1980). Además es la segunda especie 
más antigua, luego de Glyptotherium texanum Osborn, 1903 de la que se cree evolucionó (Gillette \& Ray, 1981). Aunque estas dos especies tienen una distribución paleogeográfica tropical y subtropical muy similar, al parecer nunca coexistieron en ninguna fauna local conocida (Kurtén \& Anderson, 1980).

Se considera que estas especies tuvieron hábitos semiacuáticos y gustaban de cuerpos de agua tranquilas con abundante vegetación lo que concuerda con las facies lacustres de Palmares en que se hallaron los fósiles.

\section{CONCLUSIÓN}

Se describen nuevos restos de gliptodóntidos para Costa Rica y América Central que comprenden a dos subfamilias a saber: Glyptodontinae y Glyptatelinae, representadas por los géneros: Glyptotherium asociado ala especie Glyptotherium cf. arizonae Gidley, 1926 y el primer registro para América Central del género Pachyarmatherium y de la especie Pachyarmatherium leiseyi Downing \& White, 1995.

La asociación de P. leiseyi Downing \& White, 1995 y G. cf. arizonae Gidley, 1926, permiten establecer una "edad mamífero" confiable para los sedimentos lacustres de Palmares correspondiente al Blancano Tardío o Irvingtoniano Temprano (2.6 Ma-1.6 Ma).

Por otra parte, ambos géneros probablemente evolucionaron en el Norte de América del Sur de previo a su ingreso en América Central y el hallazgo de sus restos en Costa Rica permite confirmar la continuidad migratoria de estos xenarthras entre Sur América y Norte América a través de América Central luego del cierre del Istmo de Panamá.

\section{AGRADECIMIENTOS}

A los señores Alexis Matamoros y Alfredo Duncan del Departamento de Protección del Patrimonio Cultural y a Javier Solano y Rafael Chacón del Departamento de Historia Natural del Museo Nacional de Costa Rica; quienes nos asistieron en las labores de campo, recuperación y restauración del material de estudio.

A la Licda. Carmen María Solano Martínez encargada del Proceso de Planeamiento y Evaluación, Núcleo de Turismo del INA, por el apoyo logístico brindado. Esta investigación ha sido posible gracias a las contribuciones de la OTS, Estación Las Cruces; el Grupo ESCO, San José; y donantes privados.

\section{REFERENCIAS}

CARLINI, A., VIZCAÍNO, S. \& SCILLATOYANÉ, G., 1997: Armored xenathrans: a unique taxonomic and ecologic assemblage.- En: KAY, R., CIFELLI, L., FLYNN, J. \& MADDEN, R.H. (Eds.): Vertebrate Paleontology of the Miocene Honda Group, Republic of Colombia.Smithsonian Institution Press Washington: 213-226.

CISNEROS, J.C., 2005: New Pleistocene Vertebrate Fauna from El Salvador.Revista Brasileira de Paleontologia, 8(3): 239-255,

DOWNING, K. \& WHITE, R., 1995: The cingulates (Xenarthra) of Leisey Shell Pit local fauna (Irvingtonian), Hillborough County, Florida.- Bull. of the Florida Mus. of Nat. Hist. 37: 375-396.

GILLETTE， D.D. \& C.E. RAY, 1981: Glyptodonts of North America. Smithsonian Contributions to paleobiology 40: 1-225.

HOFFSTETTER, R., 1958: Xenarthra. En PIVETEAU, J. (ed.): Traité de Paleontologie. Masson \& Cie., París. VI(2): 535-636.

HULBERT, R.C., 2001: Xenarthrans. En R.C. Hulbert (ed.): The Fossil Vertebrates of Florida. University Press of Florida, Gainesville. 175-187. 
KAY, R. \& MADDEN, R.H., 1997: Mammals and Rainfall: paleoecology of the middle Miocene at La Venta (Colombia, South America). Journal of Human Evolution 32:161-199.

KURTÉN, B. \& ANDERSON, E., 1980: Pleistocene Mammals of North América.443 págs. Columbia University Press, New York.

MCKENNA, M. \& BELL, K., 1997: Classification of Mammals above the specie level.- 631 págs. Columbia University Press, New York.

LUCAS, S.G., WILLIAMSON, T.E. \& SOBUS, J., 1993: Plio-Pleistocene stratigraphy, paleoecology and mammalian biochronology, Tijeras Arroyo, Albuquerque area, New Mexico.- Geology, 15: 1-8.

MORGAN, G. S. \& LUCAS, S. G., 2003: Mammalian Biochronology of Blancan and Irvingtonian (Pliocene and Early Pleistocene) Faunas from New Mexico.En: LAWRENCE, J. \& FLYNN, J. (Ed): Vertebrate fossils and their context:
Contributions in Honor of Richard $\mathrm{H}$. Tedford.- Bull. Amer. Mus. Nat. Hist. 270: 269-320.

VALERIO, A.L., LAURITO, C.A. \& GÓMEZ, L.D., en prensa: Un Gliptodonte (Xenarthra, Cingulata) de la Localidad de Chachagua, Provincia de Alajuela, Costa Rica.- Rev. Geol. Amér. Central, 32: 61-63.

VIZCAÍNO, S., RINDERKNECHT, A. \& CZERWONOGORA, A., 2003: An Enigmatic Cingulata (Mammalia: Xenarthra) from the Late Miocene of Uruguay.- Journal of Vertebrate Paleontology, 23(4):981-983.

WEBB, S.D., 1974: Chronology of Florida Pleistocene Mammals.- En WEBB, S.D. (Ed.): Pleistocene Mammals of Florida. The University of Florida Press, Gainesville: 5-31.

WEBB, S., MORGAN, G., HULBERT, R., JONES, D., MACFADDEN, B.\& MUELLER, P., 1989: Geochronology of a rich early Pleistocene vertebrate fauna, Leisey Shell Pit. Tampa Bay, Florida.Quaternary Research, 32(1): 96-110. 